\section{On the sombre view of AIDS}

SIR - "The sombre view of AIDS" by Malcolm Rees (Nature 326, 343; 1987) presents a more pessimistic picture than hitherto described, based on a data analysis in which the incubation period for AIDS (acquired immune deficiency syndrome) is estimated to have a mean of 15 years and standard deviation 5 years. He does not state how the estimation is performed. Barton (Nature 326, 734; 1987) pointed out that the data analysis seemed to be incorrect and that the mean is probably much more like 5 years. His analysis is based on a more nonparametric approach (Rees assumed that AIDS incubation time is normally distributed) and takes some account, but not full account, of the fact that the data are truncated (see below). The 5-year estimate is itself somewhat ad hoc; it is not given by a formula. I also believe the data analysis of Rees to be incorrect, and I should like to explain how a proper analysis based on the normality assumption, but taking full account of truncation, can be carried out. Such an analysis entails a formula for the mean and standard deviation. It confirms Barton's observation that the data support a mean value of 5 years, a number which is similar to other estimates of mean incubation time (Peterman, T.A. et al., J. Am. med. Ass., 254, 2913-2917; 1985), and, therefore, also supports a considerably less pessimistic picture.

The data consist in part of the time from infectious blood transfusion to AIDS diagnosis for each of the 144 patients with blood-tranfused AIDS reported to the Centers for Disease Control (CDC) during the period 1978-84(5). From these data, the mean and standard deviation of the probability distribution of AIDS incubation times are to be estimated. The author suggests that the estimation be performed under the assumption that this distribution is normal.

Each one of the 144 people reported to the CDC and transfused in 1978 can have an incubation period of no more than 7 years, which I call the truncation time associated with 1978. Similarly, each of the 144 persons transfused in 1979 can have an incubation period of no more than 6 years, the truncation time associated with 1979. In general, with each of the years a person in the sample could have become infected, there is an associated truncation time. Under the normality assumption, the probability distribution of the incubation time of a sampled individual is a truncated normal distribution (Continuous Univariate Distributions - 1, Johnson, N.L. \& Kotz, S., Houghton Mifflin Co., 1970) with truncation time determined by the year the individual was transfused and which is described above. Consequently, among the sampled individuals, the distribution of the incubation time is individualspecific (the truncation times vary between individuals, but are known constants). But these distributions share the two parameters of interest, the mean and standard deviation of the underlying normal distribution for the general population of infected individuals. One may fit these distributions to the data the author presents in his Table 1 using the classical statistical procedure of maximum likelihood (Theoretical Statistics, Cox, D.R. \& Hinkley, D.V.; Chapman and Hall, 1974). The incubation times are reported in years, rather than on a "continuous" scale, giving rise to many tied observations. However, the truncated normal continuous scale can be appropriately discretized by taking the probability that the reported incubation time for a sampled individual is $i$ years to be the difference in the values of the individual's (cumulative) truncated normal distribution function at years $i$ and $i-1$. Then the product of these probabilities, taken over all sampled individuals, is maximized with respect to the two underlying parameters, mean and standard deviation. The values of these parameters giving the maximum are the desired estimates. Using this approach, the mean and standard deviation parameters are estimated to be 4.51 and 1.56 years, respectively. The standard error estimates for these parameters are 0.468 and 0.176 respectively (as obtained from the inverse estimated information matrix)

Department of Laboratory Medicine,

School of Medicine,

University of California, San Francisco, San Francisco, California 94143, USA

\section{Defending 'pragmatypes'}

SIR-Endrödy-Younga (Nature 327, $664 ; 1987)$ rejects my proposal for 'pragmatypes' (Nature 326, 251; 1987) first, with reference to Recommendation $75 \mathrm{E}$ of The International Code of Zoological Nomenclature (1985 edition). This is unacceptable not only because a Neotype, by definition $^{1}$, replaces a lost type (as opposed to an existing type) but because Recommendation $75 \mathrm{E}$ is itself unacceptable. It is so worded that, taken at its face value, it succeeds in being an impediment to science. This is discussed elsewhere ${ }^{2}$.

Second, Endrödy-Younga is alarmed by the prospect that a pragmatype may, with advance in knowledge, itself become an impediment and have to be replaced by a subsequently designated new pragmatype. Why is this a problem? Types are designated in order to assist the resolution problems of nomenclature that may arise with advance in taxonomic understanding.
They are not, despite the attitudes of certain museum curators, analogues of antiquities such as the Elgin Marbles. It is the advancement of science that matters not the preservation of the status of particular biological specimens.

$$
\text { R. H. L. DISNEY }
$$

Department of Zoology,

University of Cambridge,

Downing Street,

Cambridge CB2 3EJ, UK

Jeffrey, C. Biological Nomenclature (Arnold, London, 1977).

Disney, R.H.L. \& Erzinçlioğlu, Y.Z. Bull. zool. Nomen (in the press).

\section{Maturational patterns in early hominids}

SIR-In a letter to Nature, Smith ${ }^{i}$ finds that certain early hominid jaws and associated teeth show an 'ape-like' pattern in the timing of dental calcification. She concludes that these early hominids had foreshortened growth and development periods similar to modern apes. We find a number of problems with Smith's interpretations of these dental data.

First, numerous studies of human dental calcification and eruption document that no single sample's mean ages for these features adequately represent diverse values for individuals in human populations (see, for example, refs $2-7$ ). Individual tooth calcification stages may vary by as much as $3-5$ years within and between samples. Therefore, the ages assigned by Smith to the fossil teeth do not represent their actual 'dental ages' but are sample-dependent mean values. The problem is that while Smith claims to demonstrate evolutionary patterns in dental development by two approaches, "fit to standards' of apes and humans and patterns in the plots of individual teeth relative to that of the M1, we find that a similar analysis of dental calcification stages in ten modern human specimens and of the fossil specimens presented by Smith shows variable patterns depending on the human standard-mean ages used $^{2-6}$. Modern humans are 'best fit' to pongid standards and characterized by a "primitive pattern' in eight out of ten cases'. Thus the method is clearly invalid. There is no a priori method which directs the choice of one human sample's means over another to analyse these fossils, as none can be said to be more closely related to the fossil sample under study than any other.

Second, Smith misuses growth standards, confusing data on a group with that for an individual. The age of a child is not often equal to a mean age derived from individual teeth. Synchronous teeth from individual children are not of equal age as assessed from sample means (as assumed by Smith) but can differ by as much as 2.25 years $^{8}$ (see Table 1). Furthermore, pub- 